\title{
Study on the Level of Aflatoxin M1 Contamination in Raw and Processed Milk Marketed in Kathmandu Valley
}

\section{PRATAP KAFLE ${ }^{*}$, DAMODAR SEDAI ${ }^{2}$, KRISHNA PRASAD RAI ${ }^{3}$ and BISHOW BANDHU POKHAREL ${ }^{4}$}

\author{
${ }^{1}$ Department of Veterinary Medicine, University of Calgary, Canada \\ ${ }^{2}$ Department of Livestock Services, Central Region, Kathmandu \\ ${ }^{3}$ Central food Lab, Department of Food Technology and Quality Control, Babarmahal, Nepal \\ ${ }^{4}$ Department of Animal Science, University of Manitoba, Canada
}

Aflatoxin M1 (AFM1) is the principal hydroxylated AFB1 metabolite mainly present in milk. In this study the levels of Aflatoxin M1 (AFM1) in Raw and Pasteurized milk marketed in Kathmandu valley was estimated. Altogether 32 milk samples (Raw 16, Pasteurized 16) obtained from different areas of Kathmandu valley were analysed for AFM1 by Thin Layer Chromatography. The milk samples were analyzed according to the official AOAC methods, which included extraction of toxin using chloroform, clearing by silica gel column chromatography, qualitative analysis by Thin Layer Chromatography and quantification by Visual comparison of the spots. AFM1 was found in 14 (43.75\%) of milk samples examined. The levels of AFM1 in 7 (21.87\%) samples were higher than the maximum tolerance limit $(0.05 \mu \mathrm{g} / \mathrm{l})$ accepted by some European countries while none of the samples exceeded the prescribed limit of US regulations. The mean concentration of AFM1 was higher in Raw milk $(0.030 \pm 0.042 \mu \mathrm{g} / \mathrm{l})$ compared to pasteurized $(0.022 \pm 0.039)$ but the difference was not statistically significant $(p>0.05)$. This finding reflects that milk marketed in Kathmandu valley contains residual level of Aflatoxin $M 1$ and pose public health risk. Therefore, milk and milk products have to be screened for AFM1 contamination periodically.

Keywords: Aflatoxin M1, Public health, Thin Layer Chromatography, Column Chromatography

\section{Introduction}

Aflatoxins are a group of closely related heterocyclic compounds produced predominantly by two filamentous fungi, Aspergillus flavus and Aspergillus parasiticus. Recent studies have shown that some $A$. nominus and $A$. tamarii strains are also aflatoxin producing, of which A.nominus is phenotypically similar to A. flavus (Kurtzman et al., 1987; Goto et al., 1997). Aflatoxins $\mathrm{M}_{1}$ and $\mathrm{M}_{2}$ are the hydroxylated metabolites of aflatoxins $B_{1}$ and $B_{2}$ and can be found in milk or milk products obtained from livestock that have ingested Aflatoxin contaminated feed.

Aflatoxin M2 is rarer than M1 and not as toxic so it receives little interest. Aflatoxin M1 has also been isolated on highly contaminated corn samples where it occurs 1000 times lower concentration than Aflatoxin B1 (Shotwell et al., 1976). Aflatoxin M1 is chemically stable; it is not destroyed under domestic conditions such as microwave or oven heating however the stability of Aflatoxin M1 during pasteurization is in debate. Bakirci, (2001) and Henry et al., (1997) report that pasteurization has no effect whereas Deveci and Sezgin (2006) suggests that pasteurization causes a $16 \%$ decrease, hypothesizing that the decrease is due to heat treatment causing casein decomposition.

The WHO International Agency for Research on Cancer (IARC) has classified both Aflatoxin B1 and Aflatoxin M1 as carcinogenic agents to humans (IARC, 2002). Aflatoxin M1 manifests its toxic effects by linking its adverse effects with

\footnotetext{
*Corresponding author, E-mail: pratapkafle.vet@gmail.com
}

the nucleic acid in toxic ways leading to hepatotoxicity and carcinogenicity (Wong et al., 2000).

Aflatoxicosis is the name given to the disease caused by the harmful effects of Aflatoxin. There are two courses of the disease: acute and chronic. Acute Aflatoxicosis results in deaths from hepatic necrosis and liver failure. Chronic Aflatoxicosis in humans and animals are related to cancer, immune suppression, heptocellular carcinoma, Reyes syndrome, cirrhosis and kwashiorkor (Stora /et al., 1983; Bennett and Klich, 2003).

EU countries have the lowest allowable concentrations AFM1 in milk, which is $0.05 \mu \mathrm{g} / \mathrm{l}$ (Commission Regulation (EC) N. 466/2001), while other countries have legislation for this mycotoxins ten times higher, which made allowable concentrations of $0.5 \mu \mathrm{g} / \mathrm{l}$.

Behind the veil of opaque whiteness, every quart of milk may hide a potential peril to the public health. To the unaided scenes, unwholesome or dangerous milk may present exactly the same appearance as the purest and safest supply obtained. Today all over the globe the health conscious consumers are looking towards the products not only clean and pure but for the possible contamination by the residues which impart possible health hazards in long run. For this reason, many countries have regulations to control the levels of Aflatoxin $\mathrm{B} 1$ in feeds and to purpose maximum permissible levels of AFM1 in milk to reduce this risk. As milk is the main nutrient for infants and children and who are considered to be more susceptible to adverse effects of mycotoxins, the presence of 
Aflatoxin M1 in milk is a concern.

\begin{abstract}
Materials and Methods
Sample collection- Thirty two samples of Raw and Pasteurized milk were bought from different dairy collection centres and supermarkets of different areas around Kathmandu valley. Samples were collected and analysed during August to November 2011. All samples were analyzed before their expiry date.
\end{abstract}

Raw milk- Sixteen raw milk samples were collected for the study. Samples were purchased from different local small dairy collection centers from various regions of Kathmandu valley. The collection centres collects milk daily directly from the farmers and sells to the consumers without any processing.

Pasteurized milk- Sixteen samples of commercial pasteurized milk were purchased from supermarkets and local shops from the study area. Samples were from different commonly consumed brands. Packet milk from respective milk industries and were analyzed before their expiry date.

Analysis of sample- All the milk samples were analyzed by Thin Layer Chromatography (TLC) technique for the presence of Aflatoxin M1 according to the official methods given by Association of Analytical chemists (AOAC, 2000) with some modifications.

The basic procedure involved- Extraction of Aflatoxin from Milk samples, using chloroform. Clearing or cleaning up (Column Chromatography, silica gel). Qualitative estimation of Aflatoxin (Thin Layer Chromatography). Quantitative estimation of Aflatoxin M1 (In UV cabinet by visual comparison technique). Confirmatory test $\left(\mathrm{H}_{2} \mathrm{SO}_{4}\right.$ Spray test).

All 32 samples were taken in the period from September to December 2011. Method which was used to determine Aflatoxin M1 combines cleanup process with silica gel columns and TLC determination (AOAC, 2000).

Extraction- $50 \mathrm{ml}$ milk, $10 \mathrm{ml}$ of saturated salt solution (40 gm NaCl / $100 \mathrm{ml}$ water), and $120 \mathrm{ml}$ chloroform at $30^{\circ} \mathrm{C}$ in a $250 \mathrm{ml}$ separating funnel was shaken and allowed to separate for 2 minutes lower $\mathrm{CHCl}_{3}$ layer was Drained into $125 \mathrm{ml}$ Erlenmeyer flask. Centrifuge if layers do not separate (15 minutes at $2000 \mathrm{rpm})$. A $10 \mathrm{gm}$ anhydrous Sodium Sulphate was added to $\mathrm{CHCl}_{3}$ with stirring. The final fiLate was collected in a graduated cylinder, final volume of which was recorded and saved for column chromatography.

Clearing or cleaning up (Silica gel column chromatography)The column was half filled with $\mathrm{CHCl}_{3} .2$ gm silica was made gel slurry with $\mathrm{CHCl}_{3}$ and put into the column followed by adding 2 gm Sod sulphate above silica gel. Sample extract was now added and entire solution was drained through column by gravity. This was followed by washing column with $25 \mathrm{ml}$ toluene - acetic acid $(9+1)$ to remove colored compounds and with $25 \mathrm{ml}$ of hexane - ether - acetonitrile $(5+3+2)$ to remove fat. Elution of Aflatoxin M1 was done with $40 \mathrm{ml}$ $\mathrm{CHCl}_{3}$ - acetone $(4+1)$. The final volume was evaporated to dryness and the purified extract was stored in freeze or used immediately for further testing.

Thin Layer chromatography- The sample residue was dissolved in $100 \mu$ l of benzene - acetonitrile $(9+1)$, mixed well in vortex mixture. At the same time the Pre-coated TLC plate (TLC silica Gel 60, Merks, Dimensions $20 \times 20 \mathrm{~cm}^{2}$ ) were activated in hot air oven $\left(110^{\circ} \mathrm{C}\right)$ for $1 \mathrm{hr} .40 \mu \mathrm{l}$ of sample solution was spotted in one side and 4, 8, 12, 16 and $20 \mu \mathrm{l}$ M1 standard $(0.25 \mu \mathrm{l} / \mathrm{ml})$ in the same line to the other side of the plate. The plate was developed in developing chamber containing chloroform-acetone-isopropanol $(87+10+3)$. The solvent system was let to rise for about $12 \mathrm{~cm}$ in the plate.

Quantitative estimation of Aflatoxin M1 (UV cabinet by visual comparison technique)- After drying for some time the plate was viewed in UV cabinet (366 nm $\lambda$ ), Checked for the spots of the sample in same Rf value as that of standard. Comparison was done between the intensity of spots of the standard spot to that of sample visualized and noted the matching spot and the volume of standard spotted which matched to that of sample. The collected information was placed to the working formula and the level of Aflatoxin M1 was calculated in $\mu \mathrm{g} / \mathrm{L}$.

Aflatoxin M1 in $\mu \mathrm{g} / \mathrm{kg}$ or $\mu \mathrm{g} / \mathrm{l}$ is given by the formula

$$
\begin{gathered}
\text { Vst } \times \text { Cst } \times \text { Vet } \\
V m \times M \times V f / 120
\end{gathered}
$$

Where, Vst is the Volume in $\mu$ l of the AFM1 standard used which matches the nearest spot intensity to the florescence intensity of the sample. Cst is the Mass concentration in $\mu / \mathrm{ml}$ of the AFM1 standard. Vext denotes the volume in $\mu 1$ in which sample extract was dissolved used in the test. Vm represents volume in $\mu 1$ of the sample of the sample extract used for the test. $\mathbf{M}$ is the volume of milk in $\mathrm{ml}$ used for the test. $\mathbf{V f}$ is the volume in $\mathrm{ml}$ of the filtrate obtained in extraction steps. 120 comes from the volume of chloroform, in $\mathrm{ml}$, used for extraction.

Confirmatory test- The developed TLC plate was sprayed with $25 \%$ Sulphuric Acid by the help of sprayer. The color of the spot fluorescence given by the toxin, changed from bluish to yellowish blue which confirms the presence of Aflatoxin M1 in the spot (Blaney et al. 1985).

Statistical analysis- Data were analyzed by SPSS software (Version 16.0.0, Macrovision Corporation, USA). Overall prevalence was calculated using MS-Excel. Results were expressed as mean \pm standard deviation (SD) and also as minimum and maximum concentration of AFM1. Differences 
in AFM1 concentration between different types of milk were examined using one-way analysis of variance (ANOVA). Fisher Exact's test was applied to compare the means among different categories of level of AFM1 between raw and pasteurized milk samples. The differences between values were considered significant at $\mathrm{P} \leq 0.05$.

\section{Results and Discussion}

Table 1, summarizes the number of samples analyzed and the number of samples found to contain detectable levels of AFM1 contamination in Kathmandu Valley. From a total 32 samples, $14(43.75 \%)$ contained AFM1. The number of positive samples for raw and pasteurized milk was $8(50 \%)$ and $6(35.75 \%)$ respectively. Above table shows more positive samples for raw milk than that of pasteurized milk.

Table 1. Number and percent of negative and positive samples for each kind of milk

\begin{tabular}{llcc}
\hline Types of milk & $\begin{array}{c}\text { No. of } \\
\text { samples }\end{array}$ & $\begin{array}{c}\text { No of } \\
\text { positive } \\
\text { samples }\end{array}$ & $\begin{array}{c}\text { No of } \\
\text { negative } \\
\text { samples }\end{array}$ \\
\hline Raw Milk & 16 & $8(50 \%)$ & $8(50 \%)$ \\
$\begin{array}{l}\text { Pasteurized } \\
\text { milk }\end{array}$ & 16 & $6(37.5 \%)$ & $10(62.5 \%)$ \\
Total & 32 & $14(43.75 \%)$ & $18(56.25)$ \\
\hline
\end{tabular}

Being only the first study of AFM1 in milk marketed in Nepal, there are no any previous works to compare the contamination level of this study however lot of studies have been carried out in the Asian countries which can be works to compare with. The contamination percentage form the present study is lower than various studies by different researchers in turkey, $64.9 \%$, $84 \%$, and $72.5 \%$ respectively (Fallah et al. 2010; Aseem et al., 2011; Davoudi et al., 2011).

The similar studies in other Asian countries like India, Indonesia, South Korea yielded comparatively higher percentage of contamination, $57.5 \%$ by Nuryono et al., (2009) in Indonesia, 96.3\% by Lee et al., 2009 in South Korea, 72\% by Choudhary et al., (2007) in India, $87.3 \%$ by Shipra et al., (2004) in India.

The results revealed by this study is on the lower side than the numerous results of numerous studies abroad but it is hard to conclude the presence lower risk of AFM1 exposure in our country. This is the first study of its kind and lots more is to be revealed in the future. The comparatively smaller contamination percentage might have resulted due to the fewer sample size and less sensitive analytical method(TLC) compared to the HPLC, ELISA etc which is considered more sensitive analytical method.

Table 2. Level of Aflatoxin M1 in raw and pasteurized milk

\begin{tabular}{llll}
\hline Type of milk & Range of AFM1 & Mean \pm SD & p-value \\
\hline Raw Milk & $0.026-0.138$ & $0.030 \pm 0.042$ & \\
Pasteurized milk & $0.025-0.127$ & $0.022 \pm 0.039$ & 0.594 \\
Total & $0.025-0.138$ & $0.026 \pm 0.040$ & \\
\hline
\end{tabular}

Table 3. Different level of AFM1 contamination in raw and pasteurized milk samples

Type of milk

Frequency distribution of samples in $\mu \mathrm{g} / \mathrm{L}(\%)$

\begin{tabular}{|c|c|c|c|}
\hline & $<0.025 \mu \mathrm{g} / \mathrm{L}$ & $0.025-0.05 \mu \mathrm{g} / \mathrm{L}$ & $>0.05 \mu \mathrm{g} / \mathrm{L}$ \\
\hline Raw Milk & $8 / 16(50 \%)$ & $4 / 16(25 \%)$ & $4 / 16(25 \%)$ \\
\hline Pasteurized Milk & $10 / 16(62.5 \%)$ & $3 / 16(18.75)$ & $3 / 16(18.75 \%)$ \\
\hline Total & $18 / 32(56.25 \%)$ & $7 / 32(21.87 \%)$ & $7 / 32(21.87 \%)$ \\
\hline p- value & $>0.05$ & $>0.05$ & $>0.05$ \\
\hline
\end{tabular}


Table 2, shows the level of Aflatoxin M1 in raw and pasteurized milk samples. In total the level of AFM1 was found in concentrations ranging from 0.025 to $0.138 \mu \mathrm{g} / \mathrm{L}$. $($ Mean $=0.026 \pm 0.040 \mu \mathrm{g} / \mathrm{L})$. The mean value of raw milk is $0.030 \pm 0.042$ which is larger than the mean value of pasteurized milk. However, this difference was found to be insignificant by the one way analysis of variance $(\mathrm{p}>0.05)$.

From Table 3, it can be inferred that all positive samples were within the tolerance limit $(0.5 \mu \mathrm{g} / \mathrm{L})$ determined by USA regulations. However, 7 samples $(21.87 \%$ of the positive samples) contained concentrations above $0.05 \mu \mathrm{g} / \mathrm{L}$ which is the tolerance limit adopted by the European Community and Codex Alimentarius Commission for liquid milk and processed milk products (CAC, 2001; Creppy, 2002).

The lowest concentration detected by the employed method of analysis was $0.025 \mu \mathrm{g} / \mathrm{L}$. A total of 18 samples were detected negative. However, the possibility of these samples containing AFM1 can't be ruled out as the negative samples don't necessarily mean the concentration level $0 \mu \mathrm{g} / \mathrm{L}$. These samples may contain AMM1 which was not detected by the test. $43.75 \%$ of the tested samples contained AFM1 in the detectable level. 7 (21.87\%) of the samples contained the level of AFM1 between the range $(0.025-0.05 \mu \mathrm{g} / \mathrm{L})$ which can be considered safe. Similarly $7(21.87 \%)$ of the tested samples exceeded the tolerance limit by EU $(>0.05 \mu \mathrm{g} / \mathrm{L})$. Among the exceeded samples the number of raw samples was high 4 (25\%) compared to pasteurized 3(18.75\%). However, these findings are proved insignificant statistically by the fisher exact's test as $\mathrm{P}>0.05$. The variation in the concentration of AFM1 between raw and pasteurized sample was proved insignificant by the statistical analysis, which supports the findings given by Bakirci, (2001) and Henry et al., (1997) indicating there is no effect of pasteurization in stability of Aflatoxin M1.

Keeping the results in mind it should be noted that pasteurization by no way renders milk completely safe. The threat of mycotoxins contamination which is a concern of serious public health is still prevalent although one may feel the milk is completely safe for consumption.

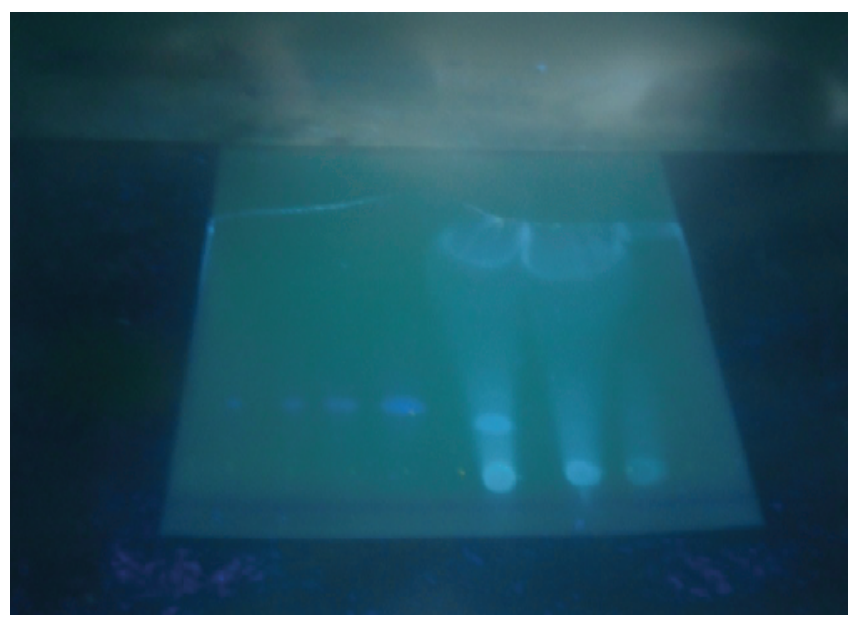

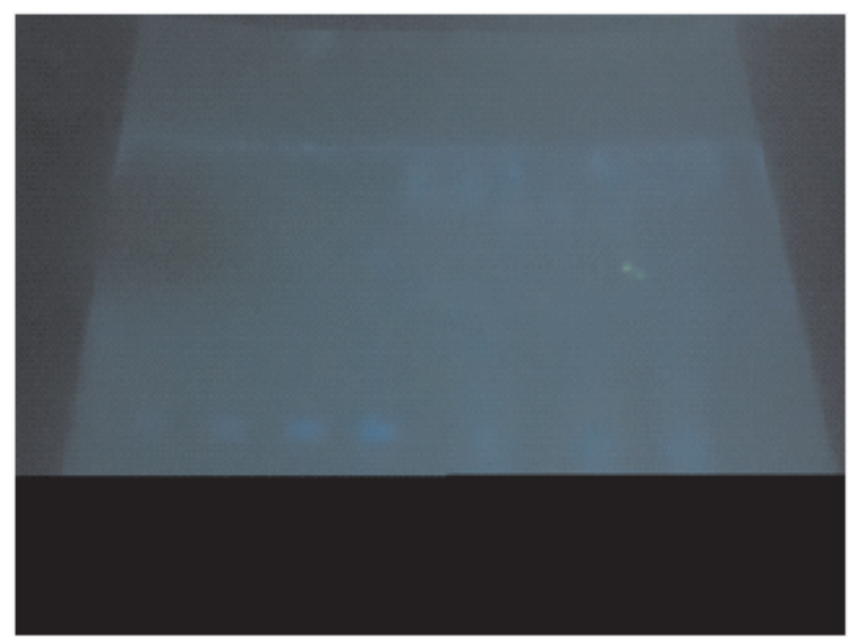

Figure 1. Plates showing positive result under UV light

\section{Conclusion}

AFM1 concentration of milk and milk products is potentially a serious public health problem as all age groups. For this reason, milk and dairy products have to be inspected and controlled continuously for AFM1 contamination. Where concentrations are unacceptably high, careful investigation of feedstuffs for contamination by AFB1 must be made, the reason for this established and the cause eliminated.

The storage mechanism of the concentrated feed is not well developed in Nepal and people do not care about the storage methods as a result of which these feed easily grow the fungus producing mycotoxins in them as a result of which there is always the possibility of milk being contaminated with the metabolised mycotoxins, mostly AFM1. It is important to maintain control and to apply an ideal recommended limit to minimize the health hazard from Aflatoxin M1 contamination in milk which it can be used by infants and children. About this, governments have responsibility for making regulations to protect consumers against harm arising from chemical in milk. Government and producer must apply some methods and plans for prevention and control of Aflatoxin M1 in milk and dairy products. About this, application of the Good Agricultural Practices (GAP) and Good Veterinary Practices (GVP) by agriculture and also the Hazard Analysis and Critical Control Point (HACCP) system as a draft code of practice for preharvest and postharvest control of dairy cow's feed and in milk and dairy products processing is effective. (Kamkar et.al., 2011). Precautions must be taken in the storage of feed commodities. Low moisture content, low temperature and low humidity conditions should be maintained during storage because these depress the fungus growth and thus eliminate Aflatoxin contamination. Responsibility for Aflatoxin M1 control in milk and dairy products lies with all participants in the production process, from farmers through to consumers. 
Analysis of Aflatoxin at $\mu \mathrm{g} / \mathrm{L}$ or $\mathrm{kg}$ level needs high tech. laboratories equipped with highly sophisticated instrumentation. Adequate number of laboratories must be established for proper analysis of aflatoxins in different foods and feed commodities and also for certification purposes, as required by the international trade. This is a first research of its kind in Nepal and there is always a scope for further research on detection of the mycotoxins in dairy products and commonly consumed food by the public.

\section{Acknowledgements}

The authors gratefully acknowledge Institute of Agriculture and Animal Science, Rampur, Nepal for financial support. Food quality Laboratory, Babarmahal, Kathmandu and Central Veterinary Lab, Tripureshwor, Kathmandu are acknowledged for laboratory and other supports.

\section{References}

AOAC, (2000). Aflatoxin M1 in milk and cheese, thin-layer chromatographic method. Natural Toxins-chapter 49 (pp. 37-38). Official Methods of Analysis of AOAC International, 17th edition, volume II, AOAC International, Official Method 980.21.

Aseem E., Abbas M. and Oula A. E. (2011). A survey on the occurrence of aflatoxin $\mathrm{M} 1$ in raw and processed milk samples marketed in Lebanon. Food Control, 22: $1856-1858$

Bakirci I. (2001). A study on the occurrence of aflatoxin M1 in milk and milk products produced in Van province of Turkey. Food Control, 12: 47-51.

Bennett, J. W., and Klich, M. 2003. Mycotoxins. Clin. Microbiol. Rev. 16(3):497-516.

Blaney B. J., Cannole, M. D., Hill, M.W.M.(1985). Aflatoxicosis. Australian Standard Diagonostic Techniques For Animal Diseases. No. 34.

Choudhary., P.L., Sahu., C and Sandey K. (2007). Aflatoxin M1 in Milk and Milk Products in Different Localities of Chhattisgarh. Indian Journal of Dairy Science. Vol: 60 , No. 5.

Codex Alimentarius Commissions(CAC). 2001. Comments submitted on the draft maximum level for Aflatoxin M1 in milk. Codex committee on food additives and cotaminants 33rd sessions, Hauge, The Netherlands.

Creppy E .E. (2002). Update of survey, regulation and toxic effects of mycotoxins in Europe Toxicology Letters Vol. 27, pp. 19-28, ISSN 0378-4274.

Davoudi Y., Yagoob, G. ( 2011). Survey on Contaminated Raw Milks with Aflatoxin M1 in the Sarab Region, Iran. Research Journal of Biological Sciences 6(2): pp 89-91.

Deveci, O., Sezgin, E. (2006). Changes in concentration of aflatoxin M1 during manufacture and storage of skim milk powder. Journal of Food Protection, 69, 682- 685 .
Fallah A. A. (2010). Assessment of aflatoxin M1 contamination in pasteurized and UHT milk marketed in central part of Iran. Food and Chemical Toxicology, 48, 988-991.

Goto T., Peterson S. W., Ito Y. and Wicklaw D. T. (1997). Mycotoxin producing ability of A.tamarii. Mycotoxins, No. 44, pp. (17-20)

Henry S., Bosch F.X., Bowers J.C., Portier C.J., Petersen B. J., Barraj L. (1997). Aflatoxins (WHO Additives, series 40.) Joint Expert Committee on Food Additives (JECFA).

IARC (1993). Monograph on the Evaluation of Carcinogenic Risk to Humans. Some Naturally Occurring Substances: Food Items and Constituent Heterocyclic Aromatic Amines and Mycotoxins (Lyon: IARC).

IARC (2002). Some Traditional Herbal Medicines, Some Mycotoxins, Naphthalene and Styrene. Summary of data reported and evaluation, IARC Monographs on the Evaluation of the Carcinogenic Risk to Humans. Vol. 82. International Agency for Research on Cancer, Lyon, France.

Kamkar, A. (2011). A study on the occurrence of aflatoxin M1 in raw milk produced in Sarab city of Iran. Food Control 16 (7), 593-599.

Kurtzman, C. P., Horn, B. W. and Hesseltine, C. (1987). A nominus, a new aflatoxin producing species related to A. flavus and A. Tamarii. Antonnie Van Leewenhoek, No. 53: (147-158)

Lee J. E., Kwak B. M., Ahn J. H. and Jeon T. H. (2009). Occurrence of aflatoxin M1 in raw milk in South Korea using an immunoaffinity column and liquid chromatography. Food Control 20 (2): 136-138.

Nuryono N., Agus A., Wedhastri S., Maryudani Y. B., Sigit Setyabudi, F M. C., Bohm, J. and Razzazi-Fazeli, E. (2009). A limited survey of aflatoxin M1 in milk from Indonesia by ELISA. Food Control 20 (8), 721-724.

Shipra, R., Premendra, D. D., Subhash, K. K., and Mukul, D. (2004). Detection of Aflatoxin M1 contamination in milk and infant milk products from Indian markets by ELISA. Food Control, 15(4),287-290.

Shotwell, O.L., Goulden, M.L. and Hesseltine, C.W. (1976). Aflatoxin M1.Occurrence in stored and freshly harvested corn. Journal of Agriculture and Food Chemistry,

Stora, C., Dvorackova, I. and Ayraud, N. (1983). Aflatoxin and Reye's syndrome. J. Med. 14(1):47-54.

Wong, N., Lai, P., Pang, E., Fung, L.-F., Sheng, Z. Wong, V., Wang, W., Hayashi, Y., Perlman, E., Yuna, S., Lau, J.W.-Y. \& Johnson, P.J. (2000). Genomic aberrations in human hepatocellular carcinomas of differing etiologies. Clin. Cancer Res., 6, 4000-4009. 